\title{
Transition of oscillatory floating half zone convection from Earth's gravity to microgravity
}

\author{
Y. L. YAO, J. Z. SHU, J. C. XIE, F. LIU and W. R. HU \\ Institute of Mechanics, Chinese Academy of Sciences, Beijing 100080, China \\ and \\ A. HIRATA, S. I. NISHIZAWA and M. SAKURAI \\ Department of Chemical Engineering, Waseda University, Tokyo, 169 Japan
}

(Received 9 September 1996)

\begin{abstract}
Oscillatory features of floating half zone convection were experimentally studied by using the drop shaft facility of Japan Microgravity Center which supported microgravity period of $10 \mathrm{~s}$. Coordinated measurements including free surface deformation and oscillation, temperature and flow pattern in both 1$\mathrm{g}$ and micro-g environment were obtained. The oscillatory frequency and amplitude in micro-g condition were lower and larger than the ones in $1-\mathrm{g}$ condition, respectively. The results gave, at first time, the oscillatory features such as free surface wave in micro-g, coordinated measurements of more than two physical quantities in the micro-g, and transition of thermocapillary oscillatory convection from 1-g to micro-g. (C) 1997 Elsevier Science Ltd. All rights reserved.
\end{abstract}

\section{INTRODUCTION}

Thermocapillary convection in floating half zone is a typical subject related to the materials processings and also important in the microgravity fluid physics, and has been studied extensively since the mid 70s [1,2]. Usually, the experiments of half floating zone convection concentrate in the studies of transition from steady convection to oscillatory convection, and the typical time scale of the experiment is $10 \mathrm{~s}$. Therefore, the microgravity experiments of the onset of oscillation have been usually completed on board the space laboratory or at least the microgravity sounding rocket. However, the transition of oscillatory thermocapillary convection in the liquid bridge with small typical scale of a few millimeters from earth's gravity to microgravity conditions could be completed in a typical time scale of $10 \mathrm{~s}$.

In the present paper, microgravity experiments of floating half zone convection were performed by using the drop shaft facility in the Japan Microgravity Center (JAMIC) for studying oscillatory features of thermocapillary convection. Usually, the thermocapillary convection has been experimentally studied by the temperature measurements of inserting thermocouple inside the liquid bridge or near the free surface [1-4]. Recently, some non-contacted methods of optical diagncstics for the measurement of the free surface oscillation and the onset of oscillation have been developed [5-7]; and the measurements of multi- quantities are possible. In the present paper, the temperature, flow pattern, edge of free boundary and free surface wave were measured coordinately both in earth's gravity and microgravity environment, and transition from 1-g to micro-g was obtained.

Ground-based experiments of small liquid bridge with typical scale of a few millimeters in diameter have been usually used to emphasize the thermocapillary effect in comparison with the gravity effect, and to simulate the microgravity process. However, the ground-based experiment with small Bond number of several $10^{-1}-10^{\circ}$ in order of magnitude will be still influenced by the earth's gravity, and different from the experiment in microgravity environment $[8,9]$. The results of present paper gave experimentally the comparison of 1-g and micro-g cases. On the other hand, the experiments of half floating zone showed that, the scale of liquid bridge and gravity level were sensitive for the onset of oscillation $[9,10]$. The liquid bridges on board the spacecraft or microgravity sounding rocket have relatively larger scale, for example, typically, several millimetcrs to several centimeters in diameter and cannot be persisted or be persisted with difficulty under the earth's gravity. It will be better to compare the results of thermocapillary convection with different typical scales, than to study the geometrical similarity. A drop shaft experiment, as given in the present paper, can be only limited in a small liquid bridge, and the results may be different from one of larger scale [10]. Furthermore, 
the free surface oscillation has been suggested as an important quantity of oscillation [4] and coordinated measurements in $1-\mathrm{g}$ condition discovered intrinsic connection of physical quantities of oscillatory convection [11], and extending the studies to the microgravity environment will also be important.

The influence of geometrical parameters such as the rod diameter $d_{\mathrm{c}}$ and geometrical aspect ratio $A=1 / d_{0}$ has been discussed extensively. Recent studies show that volume of liquid bridge is another critical geometrical parameter $[12,13]$ and its influence on the oscillatory features was studied by the present drop shaft experiments. Furthermore, the transition of oscillatory features from $1 \mathrm{~g}$ to micro-g environment was also studied. Thermocapillary convection is a typical dissipation system, which is greatly interested in the non-linear science and fluid physics. Thermocapillary convection in the microgravity environment may have different dissipative structure in comparison with the one in earth's gravity condition even for the liquid bridge of small typical scale. The present experiments measured the oscillatory features of same liquid bridge in $1-\mathrm{g}$ and micro-g, and comparison of the experiments with different gravity level will clearly show the influence of gravity.

The experiment method was discussed in the next section, then the transition from $1-\mathrm{g}$ to micro-g and the free surface waves, respectively, in Section 3 and Section 4. The last section was conclusion.

\section{EXPERIMENTAL METHOD}

Liquid bridge of 10 cst silicon oil was floated between two co-axial rods of same diameter of 3 or 4 $\mathrm{mm}$, and the height of liquid bridge is 1 . Temperatures were measured by thermocouples at upper rod, lower rods and two positions inside liquid bridge. The flow field was given by the method of tracer particles as usual in the most cases. The free surface wave was obtained especially by the grid mask method $\doteq$, and the variation of free surface edge of one cross-section of liquid bridge was measured by the system of microscope CCD Camera [6]. Coordinated measurement systems of half floating zone convection were shown schematically in Fig. 1, and the systems included temperature measurements by thermocouples (left), the flow pattern, free boundary edge and free surface variation (right). Two thermocouples passed through two small holes of $0.25 \mathrm{~mm}$ in diameter at the lower rod, and the top positions of thermocouples were, respectively, 0.8 and $0.3 \mathrm{~mm}$ in height above the lower rod. The centers of small holes were located at distance $0.675 \mathrm{~mm}$ from the edge of lower rod and $180^{\circ}$ angle difference in azimuthal direction. All data of measured quantities were recorded in the video tapes and floppy discs. The results show that microgravity period of $10 \mathrm{~s}$ during the drop is just enough to complete the transition process for small liquid bridge as adopted in the present experiment.

The drop capsule was divided into three levels to integrate the experimental payload; detailed description on the hardware was discussed elsewhere, for example, in [11]. Four half floating zone systems of two liquid bridges of $4 \mathrm{~mm}$ in diameter and two liquid bridges of $3 \mathrm{~mm}$ in diameter were packed in the bottom level of the capsule. Twelve video tape record sets were fixed in the middle level, and the electric and computer system were packed in the upper level. Teleoperation technique was used to adjust the temperature difference, the height and the volume of liquid bridge before the drop from commender room. The adjustment is important for the success of exper-

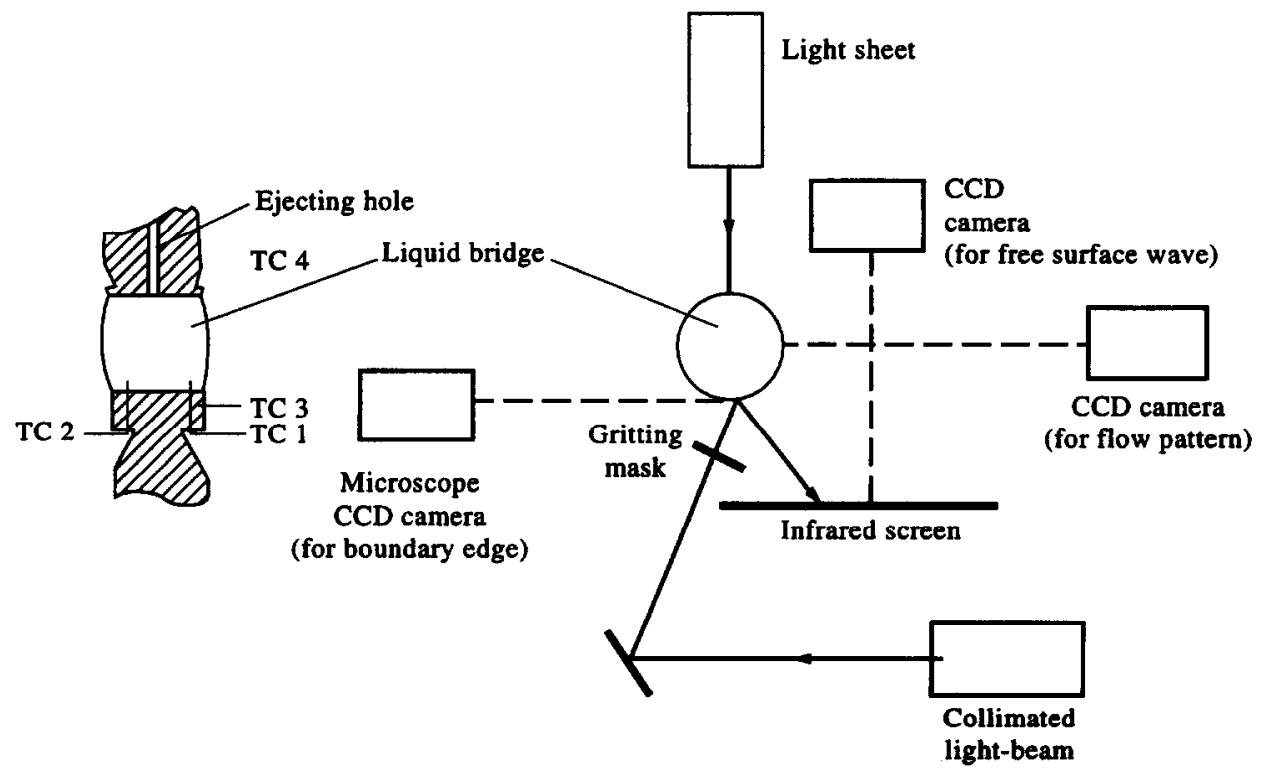

Fig. 1. The system of coordinated measurement for temperature (left), flow pattern, boundary edge and free surface wave (right), where TC denotes thermocouple. 
iments, especially for the cases of larger applied temperature difference or/and wide liquid bridge, which can persist usually for only a short period even in the ground-based experiment.

Most experiments of half floating zone study the transition process from steady to oscillatory convection. Experimental period of the transition process needs typically $10 \mathrm{~min}$ in order of magnitude for a normal heating rate, and the period of drop experiment is too short to complete the transition process. The scientific purpose of present experiments was carefully designed to be performed within $10 \mathrm{~s}$ for a quantitative microgravity experiment.

\section{TRANSITION FROM EARTH'S GRAVITY TO MICROGRAVITY}

A slender liquid bridge of $d_{0}=4 \mathrm{~mm}, d_{\mathrm{m}} / d_{0}=0.62$ and $A=0.8$ was discussed in detail in this section, and the critical applied temperature difference is $\langle\Delta T\rangle_{\mathrm{c}}=30^{\circ} \mathrm{C}$ in the $1 \mathrm{~g}$ condition. Applied temperature difference before the drop was $\langle\Delta T\rangle=60^{\circ} \mathrm{C}$ which was much larger than its critical valuc, and oscillatory states for both 1-g and micro-g condition were obtained.

The temperatures in the liquid bridge were measured by inserted thermocouples of copper-canstantan with $0.03 \mathrm{~mm}$ in diameter, and the total error included measurement error and system error was less than $0.1^{\circ} \mathrm{C}$. The radii variation of free surface boundary was measured by al microscope CCD camera system, which had relatively small view field. The shape of free surface will change obviously from $1-\mathrm{g}$ condition to micro-g condition as shown in Fig. 2, and the view field of microscopic CCD camera must be focused at the cross point of the shape cures of free surface for 1 - $g$ and micro-g condition. In this case, the radii variations for both 1-g and micro-g conditions could be recorded before the drop and during the drop. The

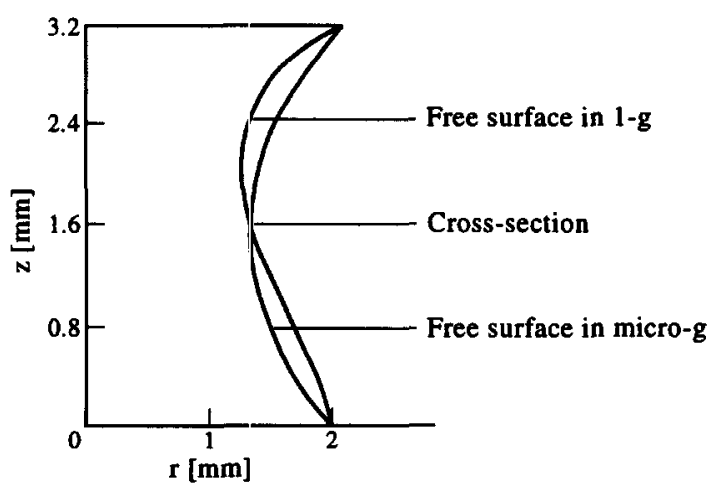

Fig. 2. Free surface shapes in 1-g condition and micro-g condition for $d_{0}=4 \mathrm{~mm}, A=0.8$ and $d_{\mathrm{m}} / d_{0}=0.6$ as an example. The cross-section locates at $(r / z)=(1.272,1.641)$, and the view field of microscopic CCD camera should be focused at the cross-section for measurement of free surface oscillations in both $1-\mathrm{g}$ and micro-g conditions. error of radii measurement in this system is 0.07 micrometer and has been discussed elsewhere.

The distributions of multi-quantities in the transition process from 1-g to micro-g could be studied by the coordinated measurement during same time evolution. The oscillatory temperatures inside the liquid bridge and the oscillation of free surface edge were coordinately obtained, for example, as given in Fig. 3. Temperature profiles at two positions had $180^{\circ} \mathrm{C}$ angle difference in azimuthal direction, and the phase difference of temperature profiles was also near $180 \leqslant$ for both $1-\mathrm{g}$ and micro-g conditions. These results imply that there may have azimuthal drift or/and oscillation in the liquid bridge. It could be seen that, transition of boundary edge just after the drop is a bit faster than the one of temperature in the liquid bridge. Similar results were obtained for other examples. These results imply that the temperature variation inside the liquid bridge is relatively a positive quantity in comparison with other active quantities, for example, the free surface oscillation. This conclusion agrees with one of coordinated measurements on the onset of oscillation in 1-g condition, where the onset of free surface oscillation is earlier than the onset of temperature oscillation [11].

It could be seen from Fig. 3 that, the frequencies of temperature and free surface oscillations were relatively higher in the $1 \mathrm{~g}$ condition than in micro-g condition, and the amplitudes of temperature and free surface oscillations were relatively small in $1 \mathrm{gg}$ condition than in the micro-g condition. The oscillatory features of thermocapillary convection depend on dissipative behaviour of the system, which is related to the gravity level. The systems of half floating zone convections in 1-g and micro-g conditions may have different dissipative features. Gravity effect may induce convection, and leads the system more disorder in comparison with the system in zero-g condition. This means that, the critical applied temperature difference $\langle\Delta T\rangle_{\mathrm{c}}$ or the critical Marangoni number $(M a)_{c}$, in 1-g condition may probably be smaller than the one in micro-g condition.

The value of critical Marangoni number depends on the heating rate $[13,15]$. The heating rate cannot be slow enough for microgravity experiment of half floating zone convection on board the sounding rocket because of the short experimental period of several minutes. Some of the space experiments show that, critical Marangoni number is at least one order of magnitude larger in the micro-g condition than in the $1 \mathrm{~g}$ condition [10], however, some others show that the critical value in $1-\mathrm{g}$ condition is larger than the one in micro-g condition. It should be pointed that, the liquid bridges in micro-g experiment have relatively larger typical scale and cannot or very difficult to be persisted in $1-\mathrm{g}$ condition. Furthermore, the small Bond number condition $B_{0} \ll 1$ requires that, the typical scale of liquid bridge should be as small as a few millimeter. The Bond number will be much larger than 1 even for a liquid bridge of $6 \mathrm{~mm}$ in 


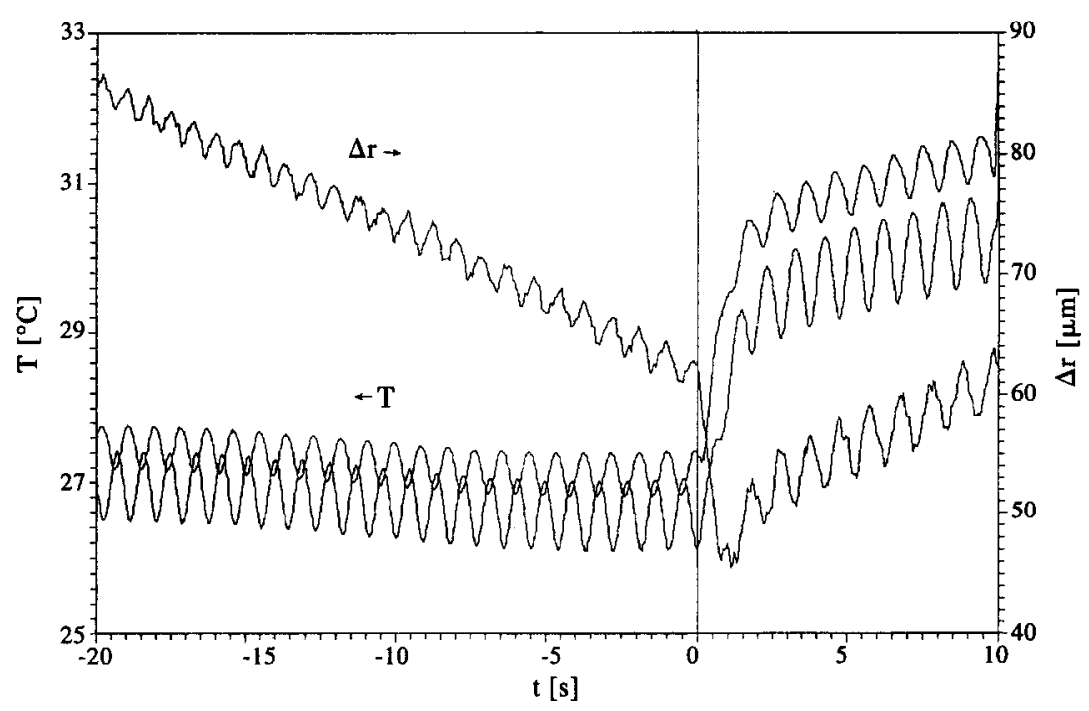

Fig. 3. Oscillatory profiles of temperature $T$ in the liquid bridge (left coordination) and free boundary edge $\Delta r$ (right coordination) transition from $1-\mathrm{g}$ to micro-g. Temperature profiles at two positions with $180^{\circ}$ angle difference in azimuthal direction in the liquid bridge, and two temperature profiles show $180^{\circ}$ phase difference. Relative radius $\Delta r=r_{\mathrm{s}}-r_{\mathrm{o}}$, where $r_{\mathrm{s}}$ and $r_{\mathrm{o}}$ are, respectively, the radius of free surface and a constant radius of free surface near the steady-state.

diameter. This implies that, the comparisons on the critical values 5 between $1-\mathrm{g}$ and micro-g will be better for small liquid bridge of a few millimeters.

The microgravity period in the drop experiment is too short to study the onset of oscillation, but the experiments can be designed to check indirectly the critical state of onset oscillation and were given by the present drop experiment. The 1-g state was adjusted to be very close to the critical state, and the applied temperature difference is very close to the critical value, either a bit larger or a bit smaller, and then the response in microgravity environment was studied. The results show that, the state in micro-g condition is also very close to the critical state if the $1-\mathrm{g}$ state is close to the critical one. An experiment gave the example of transition from oscillatory and close to the critical state in 1-g condition to non-oscillatory state in micro-g condition for slender liquid bridge as shown in Fig. 4. Another experiment gave the example of transition from non-oscillatory state and close to the critical state in 1-g condition to the oscillatory state in the micro-g condition for wide liquid bridge as given in Fig. 5. Figure 6 gave an example of transition from non-oscillation state and close to the critical state in $1-\mathrm{g}$ condition to non-oscillation state. This comparison was given for the liquid bridge with same geometrical parameters and satisfied the condition of small Bond number in both $1-\mathrm{g}$ and micro-g

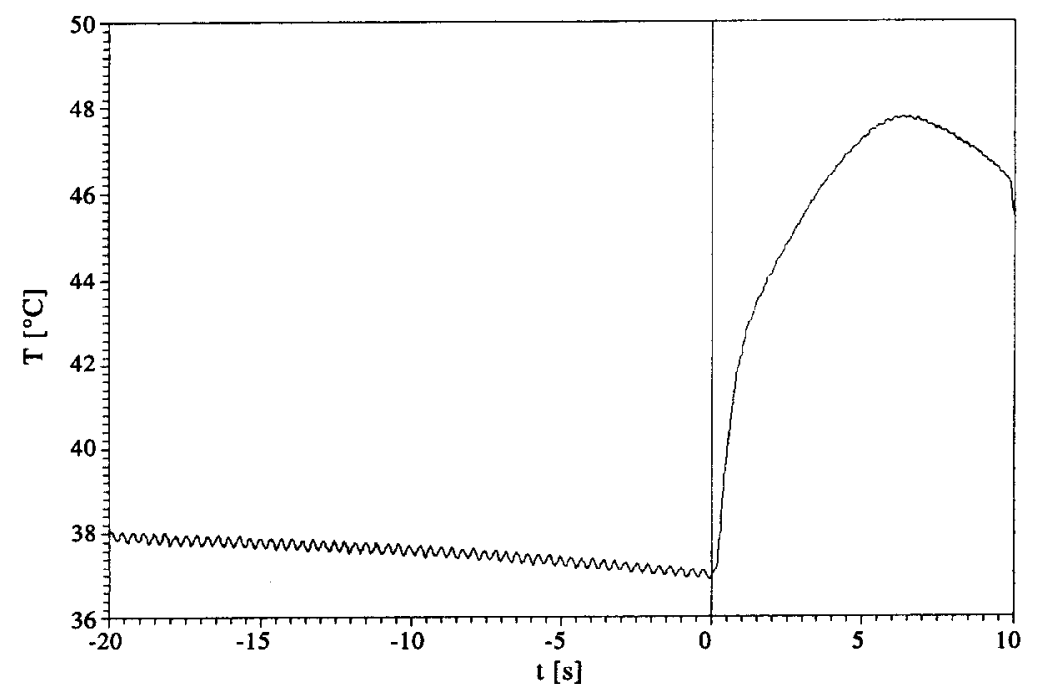

Fig. 4. Near critical state transition from oscillation in $1-\mathrm{g}$ condition to non-oscillation in micro-g condition for a slender liquid bridge of $d_{0}=3 \mathrm{~mm}, A=0.8$ and $d_{\mathrm{m}} / d_{0}=0.6$, where $\Delta T$ is $1{ }^{\circ} \mathrm{C}$ above the critical value $\langle\Delta I\rangle_{\mathrm{c}}$ in $1 \mathrm{-g}$ condition. 


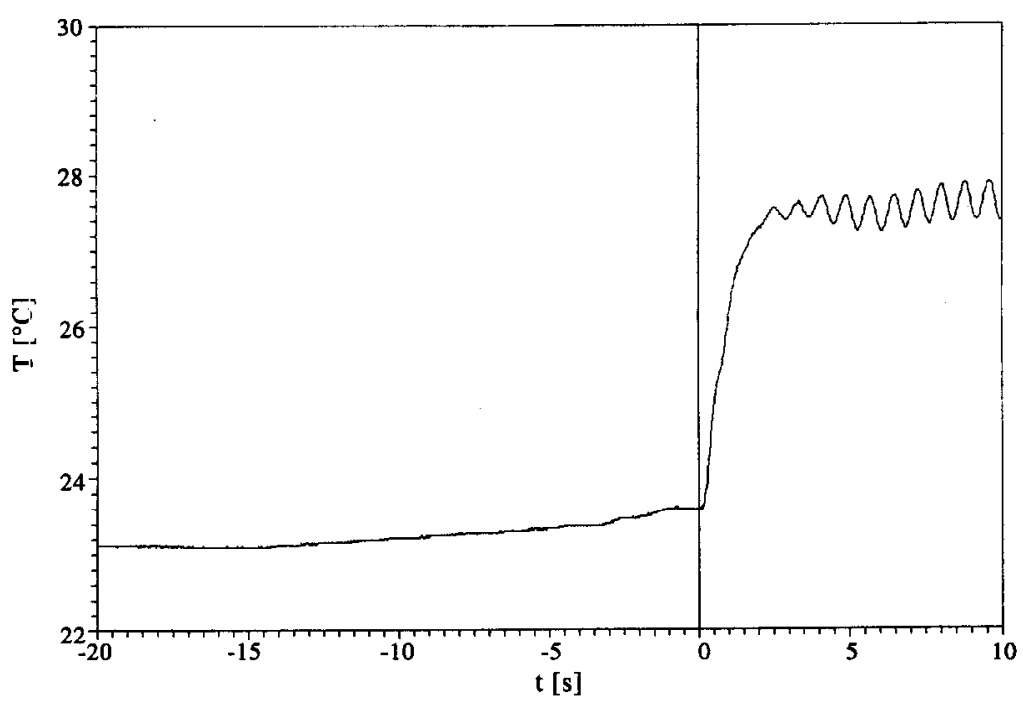

Fig. 5. Near critical state transition from non-oscillation in 1-g condition to oscillation in micro-g condition for a wide liquid bridge of $d_{0}=4 \mathrm{~mm}, A=0.8$ and $d_{\mathrm{m}} / d_{0}=0.9$, where $\Delta T$ is $1^{\circ} \mathrm{C}$ smaller than the critical value $\langle\Delta T\rangle_{\mathrm{c}}$ in 1-g condition.

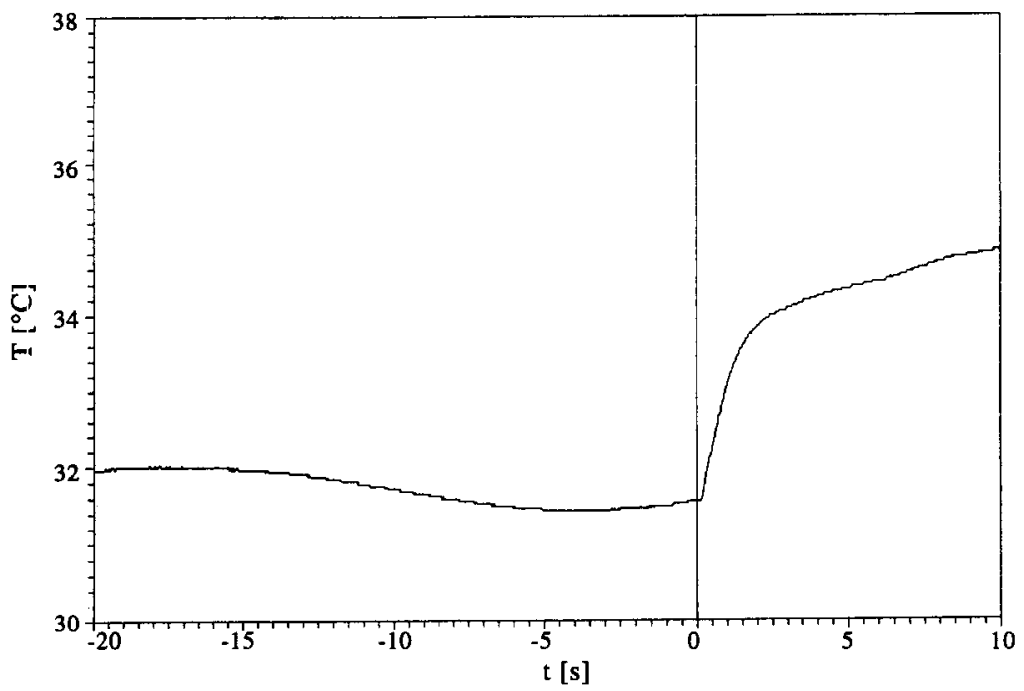

Fig. 6. Near: critical state transition from non-oscillation to non-oscillation for liquid bridge of $d_{0}=3 \mathrm{~mm}$, $A=0.8$ and $d_{\mathrm{m}} / d_{0}=0.9$, where $\Delta T$ is $1^{\circ} \mathrm{C}$ smaller than the critical value $\langle\Delta T\rangle_{\mathrm{c}}$ in $1-\mathrm{g}$ condition.

conditions. It seems that more experiments should be continued especially for different typical geometrical parameters.

\section{FREE SURFACE WAVES}

The free surface wave is generally a typical subject of hydrodynamics, and has been measured only recently for thermocapillary convection $[6,7,11]$. In the present paper, the grid mask method was used for free surface wave measurement $[7,11]$. The measurement of free surface wave was focused on a small area $0.7 \times 0.5 \mathrm{~mm}$ of free surface near the middle of the liquid bridge. The reflected image of rectangular grid could be adjusted very clear in the 1-g condition before the drop. However, the reflected grid image in micro- $\mathrm{g}$ condition is difficult to adjust during the drop period of $10 \mathrm{~s}$. In general, the free surface shape will be changed obviously after the transition from $1-\mathrm{g}$ to micro-g, and a clear grid image in the micro-g condition is not easily obtained even where there is a very clear grid image in $1-\mathrm{g}$ condition. Therefore, the evolutionary process of free surface wave transited from 1-g to micro-g condition given experimentally in the present paper is valuable for studies of thermocapillary oscillatory convection.

Several evolutions of free surface waves were obtained in both cases of $1 \mathrm{-g}$ and micro-g environment for slender liquid bridge, and the free surface configurations during one oscillatory period were given respectively, in Figs. 7 and 8 for $d_{0}=4 \mathrm{~mm}, 1 / d_{0}=0.8$ and $d_{\min } / d_{0}=0.75$ as an example. Applied tem- 

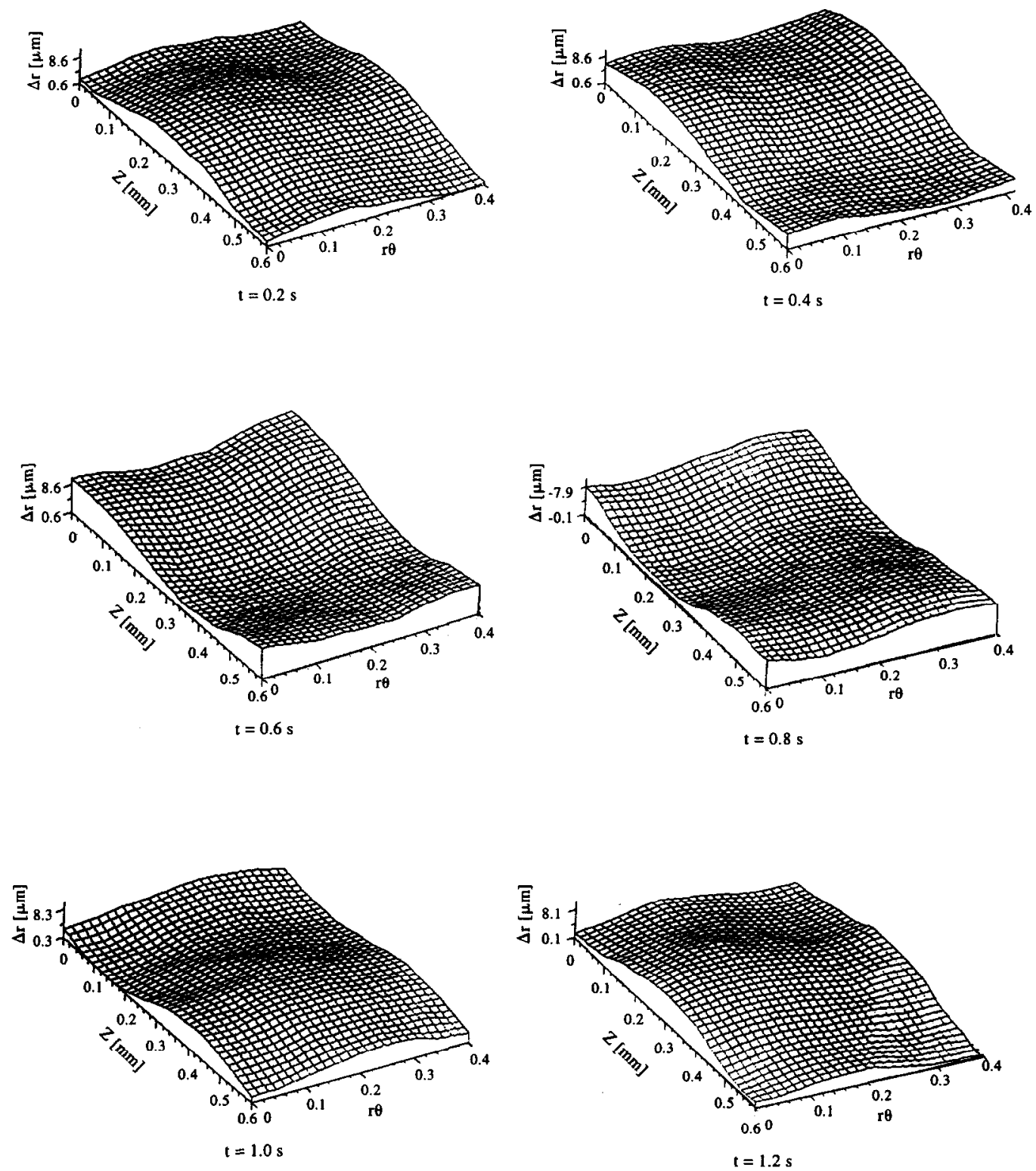

Fig. 7. The configurations of free surface wave in one oscillatory period in 1 -g condition, where $d_{0}=4 \mathrm{~mm}$, $A=0.8, d_{\mathrm{m}} / d_{0}=0.75$ and $\Delta T=60^{\circ} \mathrm{C}$.

perature difference was increased a bit in micro-g case in comparison with $1-\mathrm{g}$ condition due to the difference on the heat transfer. The oscillatory frequencies were, respectively $1.18 \mathrm{~Hz}$ for earth's gravity condition in Fig. 7 and $1.09 \mathrm{~Hz}$ for microgravity condition in Fig. 8. It could be seen that, the configurations of free surface waves for both cases are similar in general but different in details. The maximum amplitudes of free surface waves are, respectively, 6.7 micrometer for 1$\mathrm{g}$ condition and 7.6 micrometer for the case of micro-g condition. The results also show that, the longitudinal wave number is roughly $k_{\mathrm{z}} r_{\mathrm{o}}=4$ and azimuthal wave number $m \gg 1$. These results will be important to check the theoretical models. Figure 9 gave the evolutions of free surface waves in longitudinal direction of earth's gravity (a) and microgravity gravity (b). It seems that, this is an oscillatory feature of slender and small liquid bridges, shown typically by the example for both $1-\mathrm{g}$ and micro-g cases.

\section{CONCLUSIONS}

The quantitative experiments on the transition process of half floating zone oscillatory convection from $1-g$ state to micro-g state were completed by using the drop shaft facility of $10 \mathrm{~s}$ microgravity period in JAMIC. The preliminary results gave the features of thermocapillary oscillatory convections for $1-\mathrm{g}$ condition, micro-g condition, and the transition process from 1-g to micro-g state. Due to the short microgravity period of less than $10 \mathrm{~s}$, only limited experiment of fluid mechanics have been completed by 

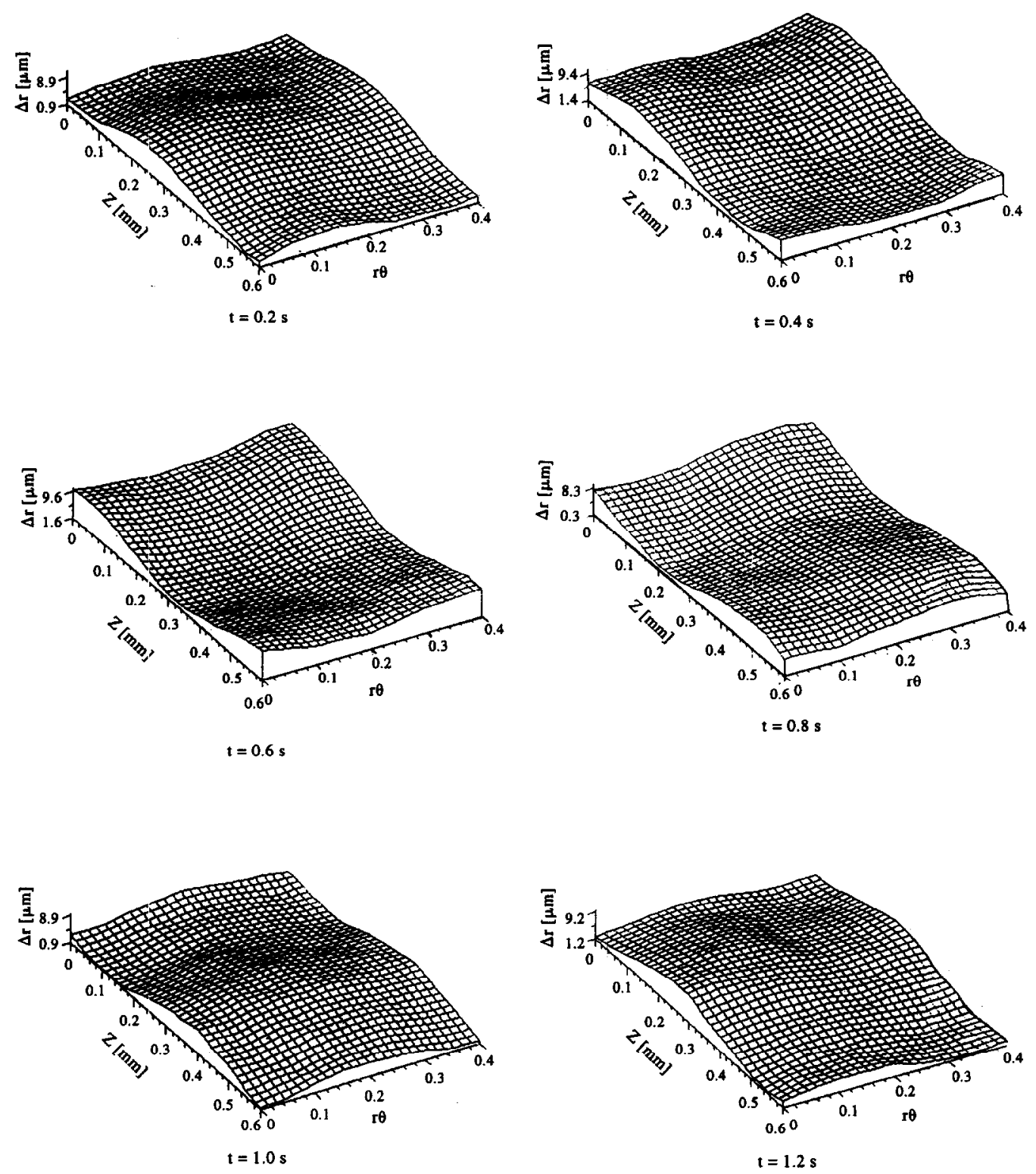

Fig. 8. The configurations of free surface wave in one oscillatory period in micro-g condition with same conditions as Fig. 6.

using the drop facility [15], and present experiment continue to use the drop shaft for research of fluid mechanics after careful design of the experimental purpose. Systematic research of the oscillatory features needs more drop experiments in the future.

Most microgravity experiments of thermocapillary convection on board the sounding rocket and spacecraft have typical scale of several millimeters to several centimeters, which are larger in comparison with the one of a few of millimeter on the ground. The present experiments study the transition process of half floating zone oscillatory convection with small Bond number for both 1-g and micro-g conditions, and experiments of small liquid bridge in microgravity environment are also important. The influence and similarity of geometrical scale for thermocapillary convection are still scientific subjects which should be analyzed, and the experiments with different typical scales need to be studied.

In the present paper, some features of thermocapillary oscillatory convection were presented for the first time, for example, the free surface wave of thermocapillary convection in the microgravity environment, the transition process of half floating zone convection from 1-g to micro-g, coordinate measurement including four physical quantities ( flow pattern, temperature, boundary edge and free surface wave) in micro-g condition. The free surface waves for both $1-\mathrm{g}$ and micro-g conditions have similar features in present experiment, and it seems that the Bond number is an important parameter in determination of the onset of oscillation [9], and also the features of free 

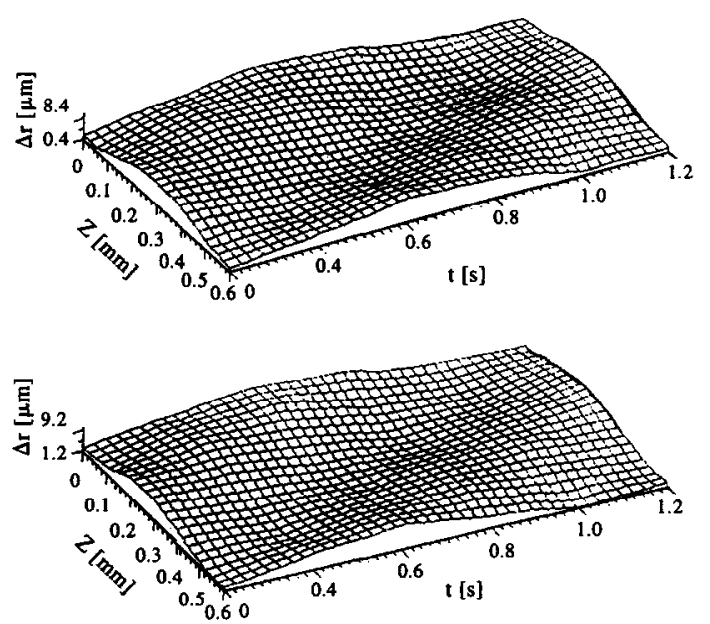

Fig. 9. The evolutions of free surface wave of longitudinal cross-sections for earth's gravity (a) and microgravity condition (b) for conditions as Figs. 7 and 8.

surface wave. These results will be beneficial for understanding the mechanisms of thermocapillary oscillatory convection.

Thermocapillary convection is different from and, in some sense, more complex in comparison with the Benard convection due to the free surface process. The free surface has non-uniform temperature distribution, which induces the convection by non-uniform surface tension, and the problem of free surface boundary will be more complicated in comparison with the problem of solid boundary. There are more critical parameters, which control the transition process of thermocapillary convection. Therefore, more experiments, in addition to the theoretical research need to be continued for studying the different aspects of the process and the influence of different parameter ranges. All these studies may shed light on the mech- anism of oscillatory thermocapillary convection. Results of present experiments are preliminary due to the limited drop times. More drop experiments should be arranged for systematic studies in the future.

Acknowledgements-Authors thank Professor Z. M. Tang for preparation of Fig. 2. The Chinese authors obtained financial support from the State Science and Technology Commission, the National Natural Science Foundation and the Chinese Academy of Sciences for the ground research and hardware development. The Waseda University applied the JAMIC drop opportunities supported by the Science and Technology Agency of Japan. Scientists of both sides thank the support from sponsors.

\section{REFERENCES}

1. Chang, C. E. and Wilcox, W. R., Journal of Crystal Growth, 1975, 28, 8.

2. Chun, C. H. and Wuest, W., Acta Astronautica, 1978, 5, 681.

3. Preisser, F., Schwabe, D. and Scharmann, A., Journal of Fluid Mechanics, 1983, 126, 545.

4. Ostrach, S., Kamotani, Y. and Lai, C. L., Physicological Chemistry Hydrodynamics, 1985, 19, 593.

5. Cao, Z. H., Xie, J. C., Tang, Z. M. and Hu, W. R., Advances in Space Research, 1991, 11(7), 166.

6. Hu, W. R., You, H. T. and Cao, Z. H., Scientia Sinica $A, 1992,35,1101$.

7. Shu, J. Z., Yao, Y. L. and Hu, W. R., Scientia Sinica A, 1993, 36, 326.

8. Tang, Z. M and Hu, W. R., Acta Mechanica Sinica, 1992, 24, 411.

9. $\mathrm{Hu}, \mathrm{W} . \mathrm{R}$. and Tang, Z. M., International Journal of Heat and Mass Transfer, 1994, 37, 1563.

10. Monti, R., Acta Astronautica, 1987, 15, 557.

11. Yoa, Y. L., Shu, J. Z., Xie, J. C., Tang, Z. M and Hu, W. R., Advance Space Research, 1995, 16(7), 218.

12. Hu, W. R. Shu, J. Z., Zhou, R. and Tang, Z. M., Journal of Crystal Growth, 1994, 142, 379.

13. Tang, Z. M. and Hu, W. R., Journal of Crystal Growth, 1994, 142, 385.

14. Xie, J. C., Wang, G. F. and Hu, W. R., Thermal Science, 1995. (in press).

15. Ostrach, S. and Pradhan, S., AIAA Journal, 1978, 16, 419. 